\title{
Genome-Wide Detection of Copy Number Variations among Diverse Horse Breeds by Array CGH
}

\author{
Wei Wang ${ }^{1,29}$, Shenyuan Wang ${ }^{1,29}$, Chenglin Hou ${ }^{1,2}$, Yanping Xing ${ }^{1,2}$, Junwei Cao ${ }^{1,2}$, Kaifeng $\mathrm{Wu}^{1,2}$, \\ Chunxia Liu ${ }^{1,2}$, Dong Zhang ${ }^{1,2}$, Li Zhang ${ }^{1,2}$, Yanru Zhang ${ }^{1,2 *}$, Huanmin Zhou ${ }^{1,2 *}$
}

1 College of Life Sciences Inner Mongolia Agricultural University, Hohhot, China, 2 Key Laboratory of Biological Manufacturing, Hohhot, China

\begin{abstract}
Recent studies have found that copy number variations (CNVs) are widespread in human and animal genomes. CNVs are a significant source of genetic variation, and have been shown to be associated with phenotypic diversity. However, the effect of CNVs on genetic variation in horses is not well understood. In the present study, CNVs in 6 different breeds of mare horses, Mongolia horse, Abaga horse, Hequ horse and Kazakh horse (all plateau breeds) and Debao pony and Thoroughbred, were determined using aCGH. In total, seven hundred CNVs were identified ranging in size from $6.1 \mathrm{~Kb}$ to $0.57 \mathrm{Mb}$ across all autosomes, with an average size of $43.08 \mathrm{~Kb}$ and a median size of $15.11 \mathrm{~Kb}$. By merging overlapping CNVs, we found a total of three hundred and fifty-three CNV regions (CNVRs). The length of the CNVRs ranged from $6.1 \mathrm{~Kb}$ to $1.45 \mathrm{Mb}$ with average and median sizes of $38.49 \mathrm{~Kb}$ and $13.1 \mathrm{~Kb}$. Collectively, $13.59 \mathrm{Mb}$ of copy number variation was identified among the horses investigated and accounted for approximately $0.61 \%$ of the horse genome sequence. Five hundred and eighteen annotated genes were affected by CNVs, which corresponded to about $2.26 \%$ of all horse genes. Through the gene ontology (GO), genetic pathway analysis and comparison of CNV genes among different breeds, we found evidence that CNVs involving 7 genes may be related to the adaptation to severe environment of these plateau horses. This study is the first report of copy number variations in Chinese horses, which indicates that CNVs are ubiquitous in the horse genome and influence many biological processes of the horse. These results will be helpful not only in mapping the horse whole-genome CNVs, but also to further research for the adaption to the high altitude severe environment for plateau horses.
\end{abstract}

Citation: Wang W, Wang S, Hou C, Xing Y, Cao J, et al. (2014) Genome-Wide Detection of Copy Number Variations among Diverse Horse Breeds by Array CGH. PLoS ONE 9(1): e86860. doi:10.1371/journal.pone.0086860

Editor: Zhanjiang Liu, Auburn University, United States of America

Received October 29, 2013; Accepted December 13, 2013; Published January 30, 2014

This is an open-access article, free of all copyright, and may be freely reproduced, distributed, transmitted, modified, built upon, or otherwise used by anyone for any lawful purpose. The work is made available under the Creative Commons CCO public domain dedication.

Funding: Financial support was provided by the Chinese Inner Mongol Agricultural University. The funders had no role in study design, data collection and analysis, decision to publish, or preparation of the manuscript.

Competing Interests: The authors have declared that no competing interests exist.

*E-mail: yanru1964@hotmail.com (YZ); huanminzhou@263.net (HZ)

9 These authors contributed equally to this work.

- These authors also contributed equally to this work.

\section{Introduction}

Previous genomic research has suggested that single nucleotide polymorphisms (SNPs) are the main form of genome structure change [1-3]. This notion changed in 2004 when two groups of scientists published the first whole-genome maps of copy number variations in seemingly healthy individuals [4,5]. Copy number variation is a form of structure variation, which has an important influence on phenotype diversity, environmental adaptability and disease susceptibility [6-8]. Copy number variant is described as a segment of DNA ranging from 50 bp to several megabases (Mb) that is copy number variable when compared with a reference genome [9], including deletion, insertion, replication and composite multisite mutation. Several studies demonstrate that about $30 \%$ of the human genome is affected by CNVs [10]. Further research suggests that, CNV exists not only in the human genome [11-13], but also widely in other mammals (pig [14,15], cattle [16-18], sheep [19,20], mice [21,22]) and plants [23,24]. CNVs contain gene coding regions or regulatory elements and may play an important role in gene expression. The effect of CNV on phenotypic diversity on domestic animal has been confirmed
$[25,26]$. Array comparative genomic hybridization $(\mathrm{aCGH})$ is a validated method to detect the amplified or deleted genome DNA [27], and has already been used for detection of CNVs in many species $[12,15,19,20]$.

Since the 1960's, with the continuous development of agricultural mechanization, the use of horses as motive power in agriculture has gradually disappeared. Horses however did not fade from human life. In many countries, horses have become domestic animals of social and economic value [28]. Different breeds are the result of the past long-term human and natural selection. Currently, there are more than 200 breeds world-wide. Nevertheless, genetic variation of different phenotypes and biological characteristics among different horse breeds are still relatively unexplored. In 2009, one UK team reported the first high-quality draft sequence of the genome of the horses [29]. Until now, CNV analyses in horses have been performed based on aCGH array [30] and SNP array [31,32]. But the study by CGH only targeted exons and therefore was not comprehensive and did not identify $\mathrm{CNV}$ s in intergenic regions.

Low temperatures, environmental hypoxia and low precipitation are the general severe nature of the plateaus. Through a long- 

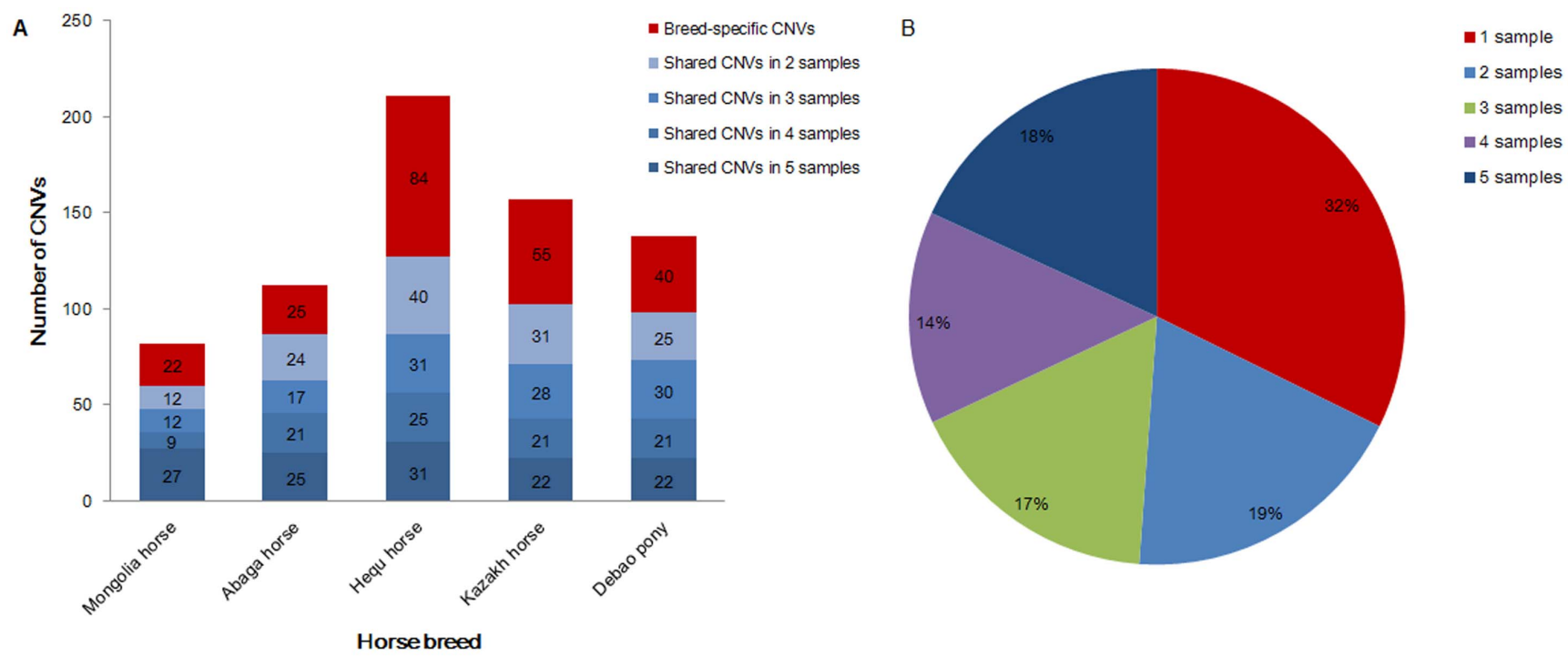

Figure 1. Distribution of CNVs in different breeds. (A) Distribution of breed-specific and shared CNVs among diverse horse breeds. The red represents breed-specific CNVs. The blue represents shared CNVs, and the color from light to dark indicates CNVs shared between 2, 3, 4 and 5 samples. (B) Percentage of CNVs shared among samples. The red represents 1 sample. The blue represents 2 samples. The green represents 3 samples. The purple represents 4 samples. The dark blue represents 5 samples.

doi:10.1371/journal.pone.0086860.g001

term evolution, Mongolia horse, Abaga horse, Hequ horse, Kazakh horse and other highland horses have adapted to live in these rigorous conditions. They could thrive normally in the highlands. While Debao pony, Thoroughbred and other non plateau horses can not adapt to the plateau's environment, and in addition have many physiological and phenotypic differences (endurance, body size) from those highland horses. In this research, we investigated genome-wide characteristics of CNVs in 6 horses representing 6 distinct breeds by using the aCGH method and performed GO and KEGG analysis for the CNVs genes. This result is an important complement to the mapping of horse whole-genome GNVs and helpful to study plateau horses' adaption to the high altitude environment.

\section{Results and Discussion}

\section{CGH-chip Design and Whole-genome Identification of CNVs}

A total of 1,402,459 probes were designed. The minimum spacing of probes was $1101 \mathrm{bp}$, the maximum spacing was $182,470 \mathrm{bp}$, and the average distance between probes was $1650 \mathrm{bp}$. In total, the probes covered 75,991,642 bp across all chromosomes (Table S1 in File S1).

Array CGH was performed to identify CNVs among 6 mare horses representing 6 diverse breeds: Mongolia horse, Abaga horse, Hequ horse, Kazakh horse, Debao pony and Thoroughbred. The first four were plateau horses and the last two were not. A single Thoroughbred mare was used as the reference sample. GNVs were analysed on autosomes by comparing the ratio of signal intensities between test samples and the reference. To determine the false positive rate (FPR) of the aCGH, we carried out a self-self hybridization of the reference Thoroughbred. A stringent criterion with the threshold value of 0.5 was used to

Table 1. The number of detected CNVs with the reference of Thoroughbred.

\begin{tabular}{|c|c|c|c|c|c|c|c|}
\hline Breed & Types & Sex & CNVs & Gains & Losses & $\begin{array}{l}\text { Average size of CNVs } \\
(\mathrm{Kb})\end{array}$ & Genes \\
\hline Mongolia horse $^{1}$ & Plateau & $\mathrm{F}$ & $82(22)$ & $42(4)$ & $40(18)$ & $33.66^{\mathrm{a}}\left(12.70^{\mathrm{b}}\right)$ & $62(7)$ \\
\hline Abaga horse $^{2}$ & Plateau & $\mathrm{F}$ & $112(25)$ & $54(8)$ & $58(17)$ & $40^{\mathrm{a}}\left(15.67^{\mathrm{b}}\right)$ & $98(20)$ \\
\hline Hequ horse ${ }^{3}$ & Plateau & $\mathrm{F}$ & $211(84)$ & $91(21)$ & $120(63)$ & $39.11^{\mathrm{a}}\left(22.62^{\mathrm{b}}\right)$ & $161(61)$ \\
\hline Kazakh horse ${ }^{4}$ & Plateau & $\mathrm{F}$ & $157(55)$ & $54(13)$ & $103(42)$ & $52.08^{\mathrm{a}}\left(33.01^{\mathrm{b}}\right)$ & $134(31)$ \\
\hline Debao pony ${ }^{5}$ & Non Plateau & $\mathrm{F}$ & $138(40)$ & $61(13)$ & $77(27)$ & $47^{a}\left(16.84^{b}\right)$ & $124(28)$ \\
\hline Total & - & - & $700(226)$ & $302(59)$ & 398 (167) & 43.08 (22.39) & 518 \\
\hline
\end{tabular}

Numbers in parentheses indicate breed-specific CNV.

a, ${ }^{b} P>0.05$, no significant difference in the average size of CNVs per individual.

$P<0.05$, significant difference in CNV status between 1 and 4,2 and 4 .

$P<0.05$, significant difference between gain and loss in 3 and 4 .

doi:10.1371/journal.pone.0086860.t001 


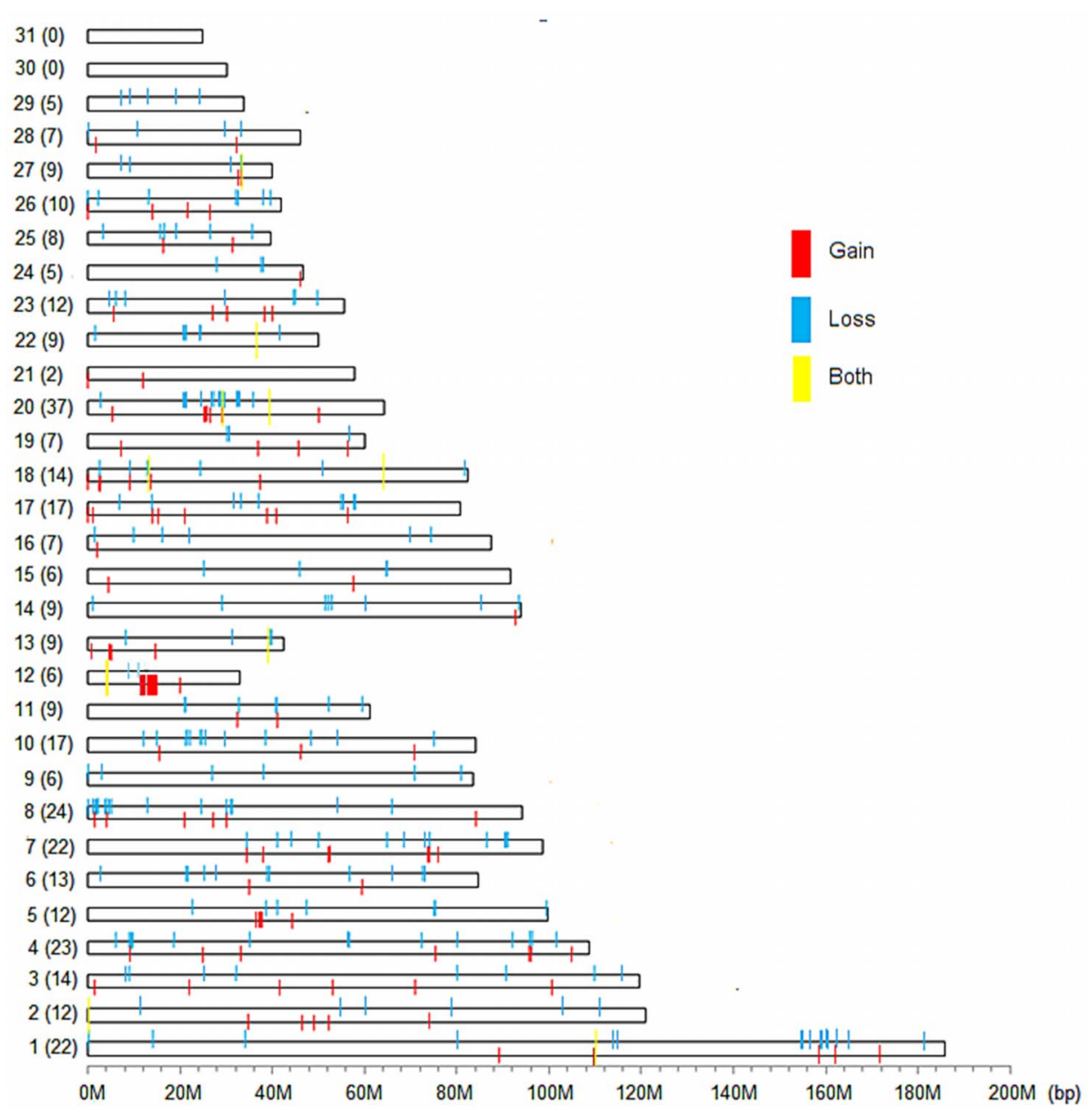

Figure 2. Distribution of CNVRs in the horse genome. Numbers in parentheses represent CNVRs. The red represents gain CNVRs. The light blue represents loss CNVRs. The yellow represents both CNVRs.

doi:10.1371/journal.pone.0086860.g002

reduce the FPR of CNV calling, and there was no GNV identified in the self-self hybridization. Therefore, we demonstrated that the result of the aCGH chip technique employed in this study to identify CNVs was reliable. In total, $700 \mathrm{CNVs}$ on autosomals were detected (Table 1 and Table S2 in File S1), including 302 gains and 398 losses. And the average number of CNVs per individual was 120, ranging from 82 to 211 , slightly less than Doan of 139.3 (2368/17, CGH analysis) [30], while significantly greater than Dupuis of 5.9 (2797/477, SNP analysis) [31] and Metzger of $5.6(4013 / 717$, SNP analysis) [32]. This might be caused by different experimental samples or detection methods of GNV. The lengths of CNVs ranged from $6.1 \mathrm{~Kb}$ to $0.57 \mathrm{Mb}$ with an average of $43.08 \mathrm{~Kb}$ and a median of $15.11 \mathrm{~Kb}$. The mean size of CNVs was low compared to the previous researches in horses $(99.4 \mathrm{~Kb}$, $229 \mathrm{~Kb}$ and $368.72 \mathrm{~Kb}$ ). CNVs were not distributed across all chromosomes, none appearing on chromosomes 30 and 31 . We defined any CNVs detected only in a single horse and not shared with another animal in this study as the breed-specific CNVs, with the number of per breed ranging from 22 to 84 . Any CNVs that identical or overlapped between two or more horses were defined as shared CNVs, the number varying from 60 to 127 (Table 1). We found that each horse shared most of their GNVs with at least another animal, and approximately $68 \%$ of the CNVs were detected in at least 2 individuals (Figure 1A, B). We didn't detect the gains and losses in the reference Thoroughbred horse, the
GNVs present in this sample would affect the results of the other breeds studied and may caused an increase in the number of shared CNVs. A total of 518 genes within or overlapped with the CNVs were identified (Table S3 in File S1), comprising about $2.26 \%$ of all the horse genes. And the number of genes within or overlapped with CNVs in each species varied from 62 to 161 .

\section{The Detailed Features of CNVRs in the Horse Genome}

After merging the overlapping CNVs, a total of 353 CNVRs were identified (Figure 2 and Table S4 in File S1) with 109 (30.88\%) CNVRs called as gains, 234 (66.29\%) called as losses and the remaining $10(2.83 \%)$ called as both (gains and losses). Comparisons between our detection results of CNVRs with recent CNVs studies showed that the number of GNVRs identical or overlapping were 93 (26.35\%), 59 (16.71\%), and 15 (4.25\%) with Doan [30], Dupuis [31] and Metzger [32], respectively (Table S5 in File S1). We found that our identified CNVRs had the highest concordance with Doan, which could be due to the same test method of CGH. In the comparison with the results of Metzger, only 15 overlapped CNVRs were found, we proposed that the difference could be due to the different research platforms, or the application of more stringent quality criteria and combination of three detection algorithms in Metzger's research. In total, 9 CNVRs were detected by all GNVs studies. The length of the 353 CNVRs ranged from $6.1 \mathrm{~Kb}$ to $1.45 \mathrm{Mb}$ with average and 
Table 2. Chromosome distribution of CNVRs in horses.

\begin{tabular}{|c|c|c|c|c|c|c|}
\hline Chr & No. of CNVRs & No. of genes & Length of CNVRs (bp) & $\begin{array}{l}\text { Length of } \\
\text { chromosomes (bp) }\end{array}$ & Percentage (\%) & $\begin{array}{l}\text { Average of } \\
\text { CNVRs(Kb) }\end{array}$ \\
\hline 1 & 22 & 59 & 1486385 & 185838109 & 0.8 & 67.56 \\
\hline 2 & 12 & 11 & 471323 & 120857687 & 0.39 & 39.28 \\
\hline 3 & 14 & 8 & 339820 & 119479920 & 0.28 & 24.27 \\
\hline 4 & 23 & 11 & 685515 & 108569075 & 0.63 & 29.81 \\
\hline 5 & 12 & 12 & 407652 & 99680356 & 0.41 & 33.91 \\
\hline 6 & 13 & 8 & 188057 & 84719076 & 0.22 & 14.47 \\
\hline 7 & 22 & 48 & 940910 & 98542428 & 0.95 & 42.77 \\
\hline 8 & 24 & 30 & 1034813 & 94057673 & 1.1 & 43.12 \\
\hline 9 & 6 & 3 & 142125 & 83561422 & 0.17 & 23.69 \\
\hline 10 & 17 & 18 & 338576 & 83980604 & 0.4 & 19.92 \\
\hline 11 & 9 & 12 & 161560 & 61308211 & 0.26 & 17.95 \\
\hline 12 & 6 & 171 & 2611353 & 33091231 & 7.89 & 435.23 \\
\hline 13 & 9 & 13 & 196374 & 42578167 & 0.46 & 21.82 \\
\hline 14 & 9 & 4 & 291428 & 93904894 & 0.31 & 32.38 \\
\hline 15 & 6 & 1 & 77346 & 91571448 & 0.08 & 12.89 \\
\hline 16 & 7 & 3 & 131240 & 87365405 & 0.15 & 18.75 \\
\hline 17 & 17 & 2 & 194530 & 80757907 & 0.24 & 11.44 \\
\hline 18 & 14 & 8 & 410207 & 82527541 & 0.5 & 29.3 \\
\hline 19 & 7 & 6 & 138162 & 59975221 & 0.23 & 19.74 \\
\hline 20 & 37 & 48 & 1526667 & 64166202 & 2.38 & 41.26 \\
\hline 21 & 2 & 3 & 78028 & 57723302 & 0.14 & 39.01 \\
\hline 22 & 9 & 4 & 265150 & 49946797 & 0.53 & 29.46 \\
\hline 23 & 12 & 3 & 173586 & 55726280 & 0.31 & 14.47 \\
\hline 24 & 5 & 1 & 89110 & 46749900 & 0.19 & 17.82 \\
\hline 25 & 8 & 10 & 271071 & 39536964 & 0.69 & 33.88 \\
\hline 26 & 10 & 8 & 251810 & 41866177 & 0.6 & 25.18 \\
\hline 27 & 9 & 6 & 176564 & 39960074 & 0.44 & 19.62 \\
\hline 28 & 7 & 4 & 469128 & 46177339 & 1.02 & 67.02 \\
\hline 29 & 5 & 3 & 38069 & 33672925 & 0.11 & 7.61 \\
\hline 30 & 0 & 0 & 0 & 30062385 & 0 & 0 \\
\hline 31 & 0 & 0 & 0 & 24984650 & 0 & 0 \\
\hline $\mathrm{x}$ & - & - & - & 124114077 & - & - \\
\hline Total & 353 & 518 & 13586559 & $2,367,053,447$ & 0.61 & 38.49 \\
\hline
\end{tabular}

doi:10.1371/journal.pone.0086860.t002

median sizes of CNVRs of $38.49 \mathrm{~Kb}$ and $13.1 \mathrm{~Kb}$, respectively. The average size of CNVRs on each chromosome ranged from 7.61 Kb to $435.23 \mathrm{~Kb}$ (Table 2). These CNVRs spaned 13.59 Mb of the horse genome and accounted for approximately $0.61 \%$ of the genome sequence. This result was shorter than Li Jiang and Joao Fadista's detection in bovine $[16,18]$, and also shorter than Doan's finding in horses [30] and Yan Li's identification in pigs [15]. We speculated that could be due to the different genetic background of the selection of animals or research platforms. And then we also assessed these CNVRs commonly affected in all experimental breeds, a total of 41 CNVRs were identified, with ranging from $4.8 \mathrm{~Kb}$ to $268 \mathrm{~Kb}$ (Table S6 in File S1).

Through the comparison of the average length of gain CNVRs $(45.92 \mathrm{~Kb})$ and loss CNVRs $(33.87 \mathrm{~Kb})$, we found gain regions had slightly larger sizes than loss regions (ANOVA not statistically significant at $P>0.05$, Table 3 ). This was similar to the previous research results that losses were under stronger purifying selection than gains [33]. The differences in GNVRs numbers per chromosome were very significant (Figure 3), ranging from 2 of chromosome 21 to 37 of chromosome 20. There was no correlation between the incidence rate of CNVRs and the length of chromosomes (Figure 4), such as the longest chromosome 1 only cotained 22 CNVRs detected but chromosome 20 (1/3 length of chromosome 1) included the largest number of 37 CNVRs. The scales of CNVRs on each chromosome were extremely different. Chromosomes 8, 12, 20, 28 had the dense CNVRs covering more than $1 \%$ of genomic sequences, and the CNVRs of chromosome 12 covered $7.89 \%$ sequences of the chromosome,especially, wheras the coverage percent on chromosome 15 was only $0.08 \%$. This demonstrated that the distribution of CNVRs on chromosomes was not uniform. We also detected 6 CNVRs with 171 genes on chromosome 12, while 37 CNVRs only containing 48 genes on chromosome 20, which appeared that chromosome 12 aberrations were in more gene dense regions. The length of 


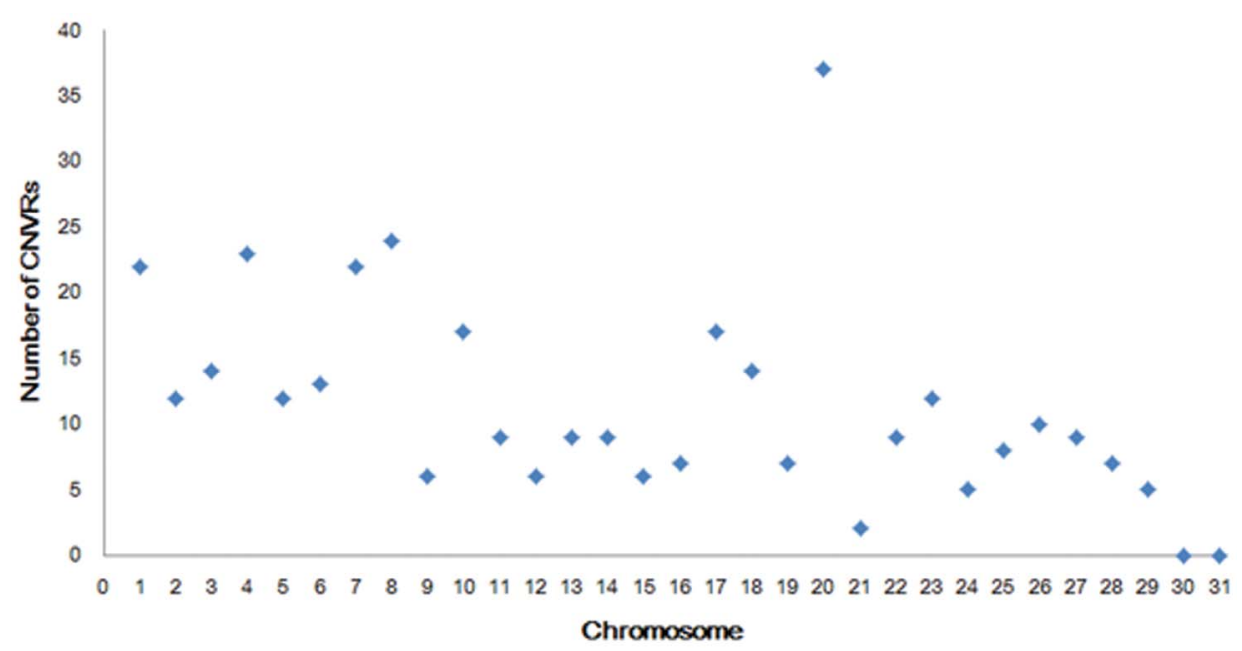

Figure 3. Chromosome distribution of the number of CNVRs. doi:10.1371/journal.pone.0086860.g003

CNVRs were divided into five regions of $1-10 \mathrm{~Kb}, 10-50 \mathrm{~Kb}, 50-$ $100 \mathrm{~Kb}, 100-500 \mathrm{~Kb}$ and $>500 \mathrm{~Kb}$ (Figure 5). Generally, the more probes used (= bigger length) the easier was to find a CNV. So the bigger the CNV was, the easier it was to detect it. While it should be noted that 291 (82.44\%) CNVRs were less than $50 \mathrm{~Kb}$, and only $2(0.57 \%)$ CNVR $>500 \mathrm{~Kb}$ in size were detected. This finding could be due to the fact that shorter GNVRs were more prevalent in horse genome, what was similar with other studies $[17,19]$.

\section{Gene Contents of Horse CNVs}

In order to analyze the genes in $\mathrm{CNVs}$ and understand the potential effects of CNVs on various biological processes, we performed functional analysis clustering of these genes affected by CNVs to understand the potential effects of CNVs on gene biotypes in horses. Two hundred and sixty five $(51.16 \%)$ protein coding genes, 231 (44.59\%) pseudo genes, 3 (0.58\%) tRNA genes and $19(3.67 \%)$ other genes were found (Figure 6). One hundred and sixty six (47.03\%) CNVRs encompassed one or more genes, however 187 (52.97\%) CNVRs didn't involve any genes. In order to determine the likely biological effects of these genes, we performed Gene Ontology (GO) analysis for these CNVs genes. Because to the horse genome was poorly annotated in the GO database, we converted all Gene Symbol IDs to the human ortholog Gene Symbol IDs (Table S3 in File S1). Functional annotation analysis was performed with the DAVID bioinformatics resources v6.7 (Accessed Mar 2013, (http://david.abcc. ncifcrf.gov/home.jsp). GO analysis revealed that GNVs genes belonged to these classes of genes that participated in olfactory receptor activity,sensory perception, cognition, G-protein coupled receptor protein signaling pathway, neurological system process, cell surface receptor linked signal transduction, plasma membrane, integral to membrane, intrinsic to membrane and other basic metabolic processes $(P<0.05$ - Table S7 in File S1). The Kyoto Encyclopedia of Genes and Genomes (KEGG) pathway analysis revealed that these genes were mainly represented in the pathway of olfactory transduction $(P<0.05$ - Table S8 in File S1). This result was similar with these findings in human, pigs and cattle.

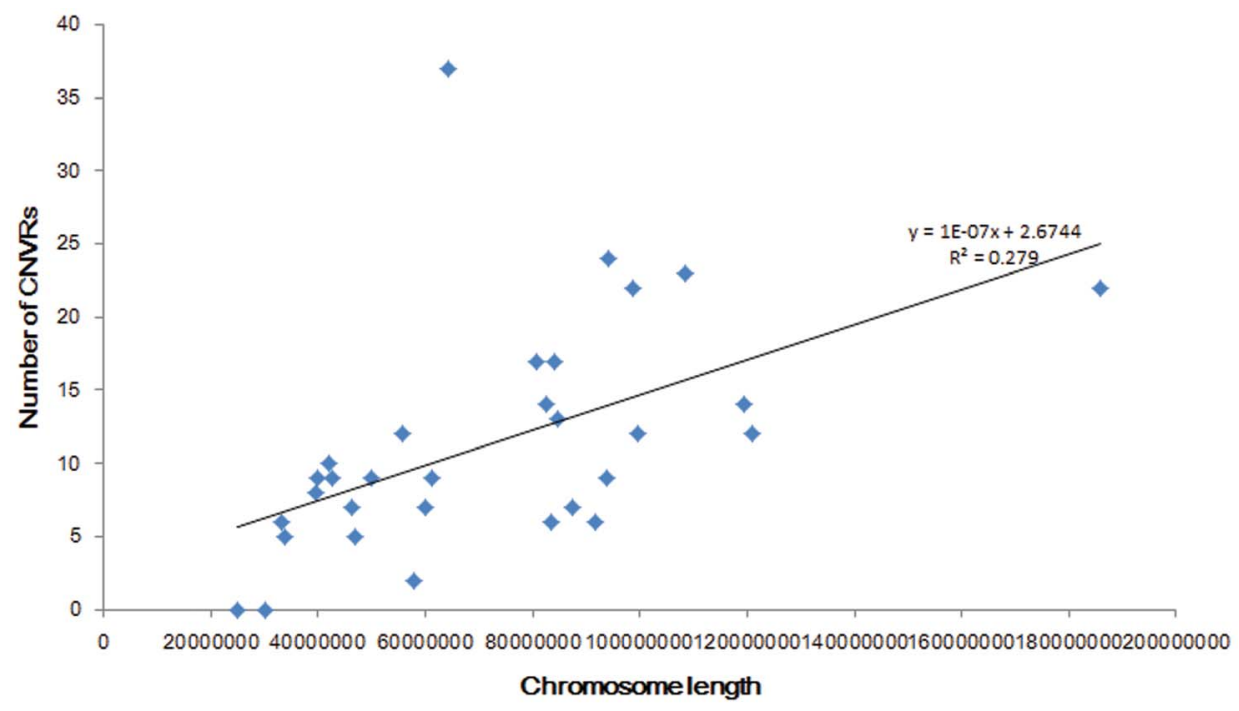

Figure 4. Correlation between the number of CNVRs and chromosome length. doi:10.1371/journal.pone.0086860.g004 


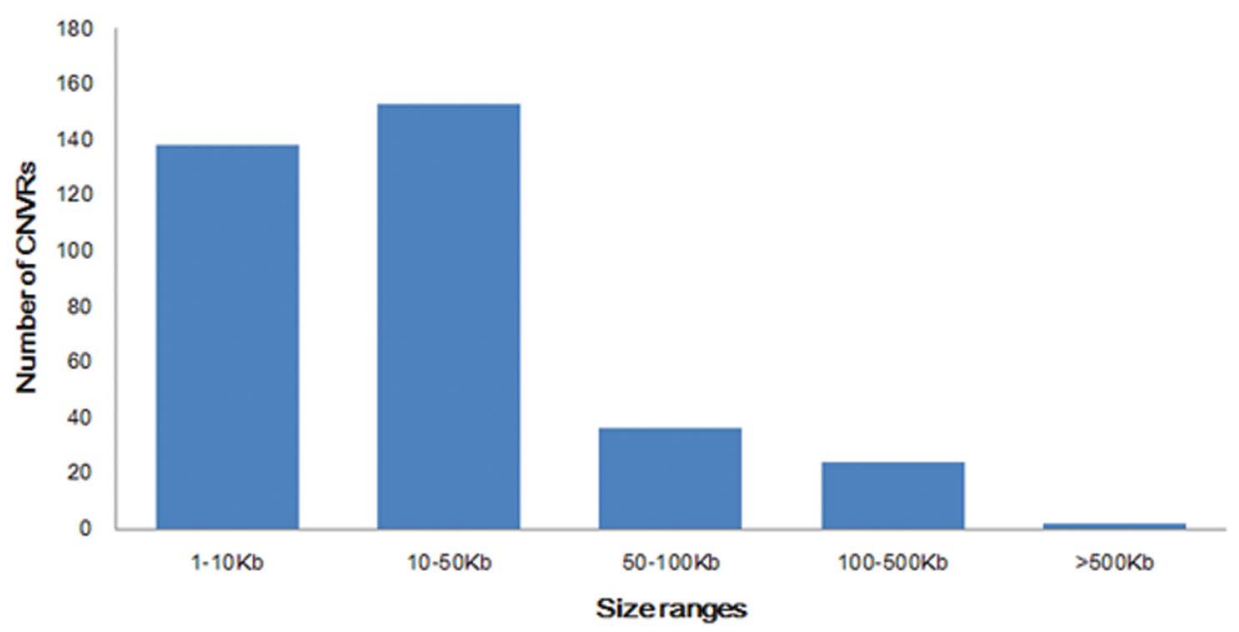

Figure 5. Size range distribution of the CNVRs detected. doi:10.1371/journal.pone.0086860.g005

Through the GO analysis for these CNVs genes, we found 7 genes (CYP4A11, GYP4X1, EIF2AK1, CYP2C18, CYP4F22, NOS2, GYP4B1) were related to the heme binding $(P<0.014)$, which correspond to the horse Gene Symbol IDs: LOC100630182, LOG100630164, LOC100062309, LOC100061400, LOC100063353, LOC100146437, LOC100629469, LOC100064176, and the majority were only in the plateau horses breed-specific CNVs (except LOC100629469) of which most existed in Hequ horse (Table 4). Hequ horse was one breed of the Chinese highland horses distributing in the eastern of Tibetan Plateau, where the altitude was above $4000 \mathrm{~m}$. They could adapt well to the harsh nature of the plateaus of low temperatures, environmental hypoxia and low precipitation through long-term evolution. And then we interrogated our data with annotations from the KEGG Pathway Database (Accessed Dec 2013, (http://www.genome.jp/kegg/ pathway.html), we found 4 genes (CYP2C18, CYP4A11, NOS2, EIF2AK1) involved in "Retinol metabolism", "HIF-1 signaling pathway", "Protein processing in endoplasmic reticulum", etc. The changes in oxygen utilization may be the key for the adaption to high altitude. From the gene ontology and genetic pathway analyses, we hypothesize that these genes in CNVs may have some relation with the adaption to the severe environment associated with plateaus in China for plateau horses. Further studies are required to assess the function of these genes in horses.

\section{DGV and OMIM Analysis}

We also queried for the horse-human ortholog genes with the Human Database of Genomic Variants (DGV, Accessed Apr 2013, (http://projects.tcag.ca/variation/) and 275 genes were found (Table S3 in File S1). In the end, to analyze whether the
CNVs affecting genes were associated with disease, the horsehuman ortholog genes were imported in Online Mendelian Inheritance in Man (OMIM, Accessed Apr 2013, (http://omim. org/), of which 96 had been associated in human disease (FSHD, Coffin-Siris syndrome, Bardet-Biedl syndrome 3, Cataract, autosomal recessive congenital 4, Blood group, ABO system, etc - Table S9 in File S1).

\section{Validation of CNVs by qPCR}

Quantitative PCR (qPCR) was performed to validate 5 GNVRs chosen from the GNVRs detected by aCGH. These GNVRs represented different status of copy number variations (gain and loss) of which all contained functional genes. Two of these GNVRs contained CYP4A11 (LOC100630182) and EIF2AK1 (LOC100062309), respectively. Among every breed, 5 or 6 different individuals were performed $\mathrm{qPCR}$ test. And results showed that, $89.69 \%$ of the qPCR results were consistent with the aCGH chip (Table S10 in File S1). Thus, qPCR results proved that the detection results of aCGH chip were credible, and also demonstrated discrepancy existed among the GNVs of different individuals of the same species.

\section{Conclusions}

In summary, we described a map of Chinese horse GNVs by a high-resolution aCGH, which was confirmed to be a valid method to detect animal genome-wide CNVs. In total, we indentified $700 \mathrm{CNVs}$, grouped into 353 CNVRs, which accounted for approximately $0.61 \%$ of the horse genome sequence. GO analysis and comparation of GNV genes among different breeds demonstrated that some genes related to heme binding could have effect

Table 3. Characteristics of the CNVRs.

\begin{tabular}{|c|c|c|c|c|c|}
\hline Status & No. of CNVRs & Length of CNVRs (bp) & Average of CNVRs (Kb) & Total length of CNVRs (bp) & Percentage (\%) \\
\hline Gains & 109 & 5005156 & $45.92^{c}$ & 13586559 & 36.84 \\
\hline Losses & 234 & 7924489 & $33.87^{c}$ & & 58.33 \\
\hline Both & 10 & 656914 & $65.69^{c}$ & & 4.84 \\
\hline
\end{tabular}

${ }^{c} P>0.05$, no Significant difference among the average of different status CNVRs

doi:10.1371/journal.pone.0086860.t003 


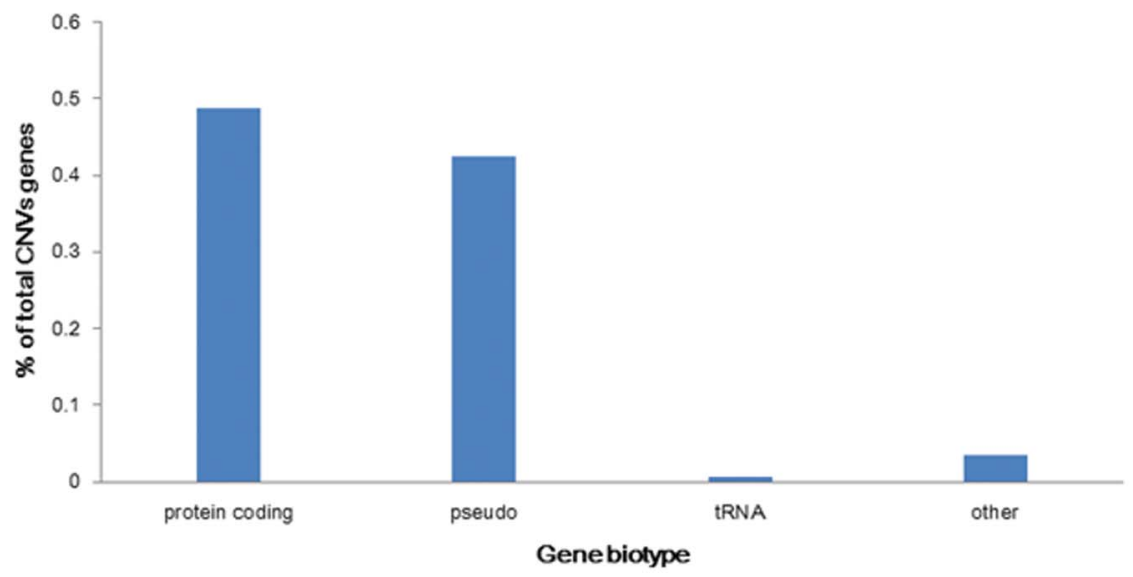

Figure 6. Percentage of gene biotypes affected by CNVs. doi:10.1371/journal.pone.0086860.g006

on the adaption to the plateau severe environment for horses. Through the validation of CNVRs by qPGR, the detection results of aCGH chip were low error rate. This study was the first report of copy number variations in Chinese horses, which will be helpful not only in mapping the horse whole-genome CNVs but also further study to the adaption to the plateau's harsh nature for plateau horses.

\section{Methods}

\section{Sample Preparation}

The whole study protocols for collection of the tissue samples of experimental individuals were reviewed and approved by the Agricultural Hall of China Inner Mongolia.

The study population consisted of Mongolia horse (Inner Mongolia, $\mathrm{n}=1$, ᄋ), Abaga horse (Inner Mongolia, $\mathrm{n}=1$, ᄋ), Hequ horse (Gansu Hequ, $\mathrm{n}=1$, )), Kazakh horse (Xinjiang Province, $\mathrm{n}=1$, ᄋ), Debao pony (Guangxi Debao, $\mathrm{n}=1$, ᄋ) and Thoroughbred (Beijing, $\mathrm{n}=1$, क). In this study, a total number of 6 individuals were chosen for CGH and they were divided into two types according to the altitude of their distributions (Table 1). We used the jugular vein blood sampling after the horse was injected with tranquillizers, blood, $15 \mathrm{~mL}$, was collected in $50 \mathrm{~mL}$ centrifuge tube with EDTA anticoagulant, and then preservated at $-80^{\circ} \mathrm{C}$. Genomic DNA was extracted from blood using AxyPrep Blood Genomic DNA Maxiprep Kit and purified by Wizard ${ }^{\circledR}$ Genomic DNA Purification Kit according to the manufacturer's instructions. The $200 \mathrm{ng}$ genomic DNA was analyzed on $1 \%$ agarose gel to ensure there were no signs of RNA contamination or degradation.

\section{Array CGH Processing and Analysis}

This study used a customized Equus caballus CGH $3 \times 1.4 \mathrm{M}$ Whole-Genome Tiling (NimbleGen), which was designed based on the Thoroughbred genome sequence published on NCBI Database in October 2007 by The Genome Assembly Team (Accessed Nov 2012, (http://www.ncbi.nlm.nih.gov/genome/ 145). The average distance between probes was $1650 \mathrm{bp}$, and through the SSAHA algorithm to select specific probes.

Labeling, hybridization, washing, array scanning and data analysis were carried out according to the NimbleGen CGH Arrays User's Guide and performed at CapitalBio Corporation (Beijing, China).

Briefly, pairs of genomic DNA (500 ng) were labeled with fluorescent dyes Gy3 (test samples) or Cy5 (reference DNA), samples were co-hybridized to Equus caballus CGH $3 \times 1.4 \mathrm{M}$ Whole-Genome Tiling, with a median probe spacing of $1650 \mathrm{bp}$.

The arrays were scanned using MS200 scanner (NimbleGen) with $2 \mu \mathrm{m}$ resolution, and fluorescent intensity data was extracted with NimbleScan 2.6 software. The hybridization control (STC, Sample Tracking Controls) were used to confirm that the correct sample was hybridized to each array.

For each spot on the array, $\log 2$ ratios of the Cy3-labeled test sample versus Cy-5 reference sample were computed. Before normalization and segmentation analysis, spatial correction was applied, which corrected position-dependent non-uniformity of

Table 4. The information of 7 genes.

\begin{tabular}{|c|c|c|c|c|}
\hline Gene Symble & Homologous Human Gene Symbl & Region & Status & Involve horse breeds \\
\hline LOC100061400 & CYP2C18 & chr1:33918586,33937152 & loss & Kazakh horse \\
\hline LOC100064176 & CYP4B1 & chr2:11618958,11710183 & loss & Hequ horse \\
\hline LOC100630164 & CYP4X1 & chr2:11618958,11710183 & loss & Hequ horse \\
\hline LOC100630182 & CYP4A11 & chr2:11618958,11710183 & loss & Hequ horse \\
\hline LOC100146437 & NOS2 & chr11:41831456,41849869 & gain & Hequ horse \\
\hline LOC100062309 & EIF2AK1 & chr13:1497390,1508926 & gain & Abaga horse \\
\hline LOC100063353 & CYP4F22 & chr21:744418,785384 & gain & Hequ horse, Abaga horse \\
\hline
\end{tabular}


signals across the array, specifically, locally weighted polynomial regression (LOESS) is used to adjust signal intensities based on $\mathrm{X}$, $\mathrm{Y}$ feature position [34]. Normalization was then performed using the q-spline method [35], compensated for inherent differences in signal between the two dyes. This was followed by segmentation using the CNV calling algorithm segMNT [36]. The segMNT algorithm identified copy number variation using a dynamic programming process that minimizes the squared error relative to the segment means, which showed better performance than the DNACopy algorithm [37]. The segments with $\mid$ mean $\log _{2}$ ratio $\mid$ $\geq 0.5$ and at least 5 consecutive probes were retained [38]. $\log _{2}$ ratio means of all probes on a segment were used to classify the segment as "gain", "unchanged" and "loss" with following criteria: $\mid \log _{2}$ ratio| $\mid<0.5$ represented "unchanged"; $\log _{2}$ ratio $\geq 0.5$ represented "gain"; $\log _{2}$ ratio $\leq-0.5$ representsted "loss". And further visualization with SignalMap software (NimbleGen).

\section{Enrichment Analysis}

Gene contents of the identified CNVRs were retrieved from the NGBI Genome Database based on the EquCab2.0 sequence assembly. Due to the horse genome being poorly annotated in the GO database, we converted all Gene Symbol IDs to the human orthologs Gene Symbol IDs. To determine their functional enrichment, we performed GO classification [39] and KEGG pathway annotation [40] of these GNVs genes with the DAVID tool [41]. We also compared these horse-human ortholog genes with the GNV related genes reported in the DGV [42]. By the end, querying for these horse-human ortholog genes with OMIM to detect CNVs affecting genes associated with disease.

\section{Quantitative PCR}

We used the qPCR method to validate CNVRs in this study. The instrumentation was Roche LightCycler ${ }^{\circledR}$ 480, analysis software was Roche LightCycler ${ }^{\circledR} 480$ Detection System. The primers were designed using the Primer Premier 5 software. A total of 5 pairs of primers covering 5 candidate CNVRs were synthesisesd plus one pair of control primers in the GAPDH gene [30] (Table S11 in File S1). All PCR primers were designed based on its reference sequence in NCBI. All qPCR reactions were performed by using SYBR Green method. The reaction system was $20 \mu \mathrm{L}$, which contained $20 \mathrm{ng}$ gDNA, $0.4 \mu \mathrm{L}(10 \mu \mathrm{M})$ of both forward primer and reverse primer, $10 \mu \mathrm{L}$ SYBR Premix Ex Taq II and water. The cycling conditions consisted of 1 cycle at $95^{\circ} \mathrm{C}$ for $5 \mathrm{~min}$, followed by 40 cycles at $95^{\circ} \mathrm{C}$ for $60 \mathrm{sec}, 60^{\circ} \mathrm{C}$ for

\section{References}

1. Sachidanandam R, Weissman D, Schmidt SC, Kakol JM, Stein LD, et al. (2001) A map of human genome sequence variation containing 1.42 million single nucleotide polymorphisms. Nature 409: 928-933.

2. International HapMap Consortium (2005) A haplotype map of the human genome. Nature 437: 1299-1320.

3. Eck SH, Benet-Pages A, Flisikowski K, Meitinger T, Fries R, et al. (2009) Whole genome sequencing of a single Bos taurus animal for single nucleotide polymorphism discovery. Genome Biol 10: R82.

4. Sebat J, Lakshmi B, Troge J, Alexander J, Young J, et al. (2004) Large-scale copynumber polymorphism in the human genome. Science 305: 525-528.

5. Iafrate AJ, Feuk L, Rivera MN, Listewnik ML, Donahoe PK, et al. (2004) Detection of large-scale variation in the human genome. Nat Genet 36: 949951.

6. Steven A McCarroll, David M Altshuler (2007) Copy-number variation and association studies of human disease. Nature Genetics 39: s37-s42.

7. Lin CH, Lin YC, Wu JY, Pan WH, Chen YT, et al. (2009) A genome-wide survey of copy number variations in Han Chinese residing in Taiwan. Genomics 94: 241-246.

8. Conrad DF, Pinto D, Redon R, Feuk L, Gokcumen O, et al. (2010) Origins and functional impact of copy number variation in the human genome. Nature 464 : $704-712$.
$40 \mathrm{sec}$, and $72^{\circ} \mathrm{C}$ for $60 \mathrm{sec}$, with fluorescence acquisition at $72^{\circ} \mathrm{C}$ in single mode. All PCRs were performed in 96-well clear reaction plates (Roche Applied Science). We used the relative quantitative method to test samples relative copy number analysis. The value of crossing thresholds (Ct) was composed of three repeated test averaging, and with normalization of control primer (GAPDH). Finally, the relative copy number of the test samples gDNA CNVR were calculated by $2^{-\Delta \Delta \mathrm{Ct}}[43]$.

\section{Data Availability}

The full data set and designs from the oligo array CGH experiments have been submitted to GEO [44] under the accession ID: GSE52504.

\section{Supporting Information}

File $\mathbf{S 1}$ File includes Tables S1-S11. Table S1: The information of aCGH chip designed by Roche NimbleGen. Table S2: The detailed features of CNVs on chromosomes identified in this study. Table S3: Gene contents of GNVs. Table S4: The detailed features of CNVRs on chromosomes identified in this study. Table S5: GNVRs identified in this study in comparison with the previous studies. Table S6: CNVRs commonly identified in all experimental breeds. Table S7: Gene Ontology (GO) analysis of CNVs genes. Table S8: KEGG Pathway analysis of CNVs genes. Table S9: The details of horse-human orthologs genes exist in OMIM. Table S10: The information of quantitative PCR. Table S11: The information of primers used in quantitative PCR.

(XLSX)

\section{Acknowledgments}

We are grateful to Yong-Hong Ren (CapitalBio Corporation) for the suggestions for this study, and to CapitalBio Corporation for the technical assistance with NimbleGen CGH analysis. We also acknowledge the horse owners (the Pony Club of Guangxi Province Debao County, the Hequ Racecourse of Gansu Province Maqu County, etc) for providing horse samples.

\section{Author Contributions}

Conceived and designed the experiments: WW YZ HZ. Performed the experiments: WW SW. Analyzed the data: WW. Contributed reagents/ materials/analysis tools: WW SW CH YX KW JC CL DZ. Wrote the paper: WW SW LZ.

9. Mills RE, Walter K, Stewart C, Handsaker RE, Chen K, et al. (2011) Mapping copy number variation by population-scale genome sequencing. Nature 470 : 59-65.

10. Database of Genomic Variants. Available: http://projects.tcag.ca/variation/. Accessed 2010 Aug 31.

11. Redon R, Ishikawa S, Fitch KR, Feuk L, Perry GH, et al. (2006) Global variation in copy number in the human genome. Nature 444: 444-454.

12. Park H, Kim JI, Ju YS, Gokcumen O, Mills RE, et al. (2010) Discovery of common Asian copy number variants using integrated highresolution array CGH and massively parallel DNA sequencing. Nat Genet 42: 400-405.

13. Alkan G, Coe BP, Eichler EE (2011) Genome structural variation discovery and genotyping. Nat Rev Genet 12: 363-376.

14. Fadista J, Nygaard M, Holm LE, Thomsen B, Bendixen G (2008) A snapshot of GNVs in the pig genome. PLoS One 3: e3916.

15. Li Y, Mei S, Zhang X, Peng X, Liu G, et al. (2012) Identification of genomewide copy number variations among diverse pig breeds by array CGH. BMC Genomics 13: 725 .

16. Hou Y, Liu GE, Bickhart DM, Cardone MF, Wang K, et al. (2011) Genomic characteristics of cattle copy number variations. BMC Genomics 12: 127.

17. Cicconardi F, Chillemi G, Tramontano A, Marchitelli G, Valentini A, et al. (2013) Massive screening of copy number population-scale variation in Bos taurus genome. BMC Genomics 14: 124. 
18. Jiang L, Jiang J, Yang J, Liu X, Wang J, et al. (2013) Genome-wide detection of copy number variations using high-density SNP genotyping platforms in Holsteins. BMC Genomics 14: 131.

19. Fontanesi L, Martelli PL, Beretti F, Riggio V, Dall'Olio S, et al. (2010) An initial comparative map of copy number variations in the goat (Capra hircus) genome. BMC Genomics 11: 639.

20. Fontanesi L, Beretti F, Martelli PL, Colombo M, Dall'olio S, et al. (2011) A first comparative map of copy number variations in the sheep genome. Genomics 97 : 158-165.

21. Watkins Chow DE, Pavan WJ (2008) Genomic copy number and expression variation within the C57BL/6J inbred mouse strain. Genome Res 18: 60-66.

22. She X, Cheng Z, Zöllner S, Church DM, Eichler EE (2008) Mouse segmental duplication and copy number variation. Nat Genet 40: 909-914.

23. Li X, Yan W, Agrama H, Hu B, Jia L, et al.(2010) Genotypic and phenotypic characterization of genetic differentiation and diversity in the USDA rice minicore collection. Genetica 138: 1221-1230.

24. Yu P, Wang C, Xu Q Feng Y, Yuan X, et al. (2011) Detection of copy number variations in rice using array-based comparative genomic hybridization. BMC Genomics 12: 372.

25. Clop A, Vidal O, Amills M (2012) Copy number variation in the genomes of domestic animals. Anim Genet 43: 503-17.

26. Fontanesi L, Beretti F, Riggio V, Gómez González E, Dall’Olio S, et al. (2009) Copy number variation and missense mutations of the agouti signaling protein (ASIP) gene in goat breeds with different coat colors. Cytogenet Genome Res 126: 333-347.

27. Sykulski M, Gambin T, Bartnik M, Derwinska K, Wisniowiecka Kowalnik B, et al. (2013) Multiple samples aCGH analysis for rare CNVs detection. Journal of Clinical Bioinformatics 3: 12.

28. Yang H, Ma YH, Li B, Dugarjaviin M (2010) Progress on horse genome project. HEREDITAS 32: 211-218.

29. Wade CM, Giulotto E, Sigurdsson S, Zoli M, Gnerre S, et al. (2009) Genome Sequence, Comparative Analysis, and Population Genetics of the Domestic Horse. Science 326: 865-869.

30. Doan R, Cohen N, Harrington J, Veazey K, Juras R, et al. (2012) Identification of copy number variants in horses. Genome Res 22: 899-907.

31. Dupuis MC, Zhang Z, Durkin K, Charlier C, Lekeux P, et al. (2012) Detection of copy number variants in the horse genome and examination of their association with recurrent laryngeal neuropathy. Anim Genet 44: 206-208.
32. Metzger J, Philipp U, Lopes MS, da Camara Machado A, Felicetti M, et al. (2013) Analysis of copy number variants by three detection algorithms and their association with body size in horses. BMC Genomics 14: 487.

33. Locke DP, Sharp AJ, McCarroll SA, McGrath SD, Newman TL, et al. (2006) Linkage disequilibrium and heritability of CNPs within duplicated regions of the human genome. Am J Hum Genet 79: 275-290.

34. Smyth GK, Speed TP (2003) Normalization of cDNA microarray data. Methods 31: 265-273.

35. Workman C, Jensen LJ, Jarmer H, Berka R, Gautier L, et al. (2002) A new nonlinear normalization method for reducing variability in DNA microarray experiments. Genome Biol 3: 1-16.

36. Molla M (2007) In PhD thesis University of Wisconsin-Madison Department of Computer Sciences. Novel Uses for Machine Learning and Other Computational Methods for the Design and Interpretation of Genetic Microarrays.

37. Olshen A, Venkatraman E, Lucito R, Wigler M (2004) Circular binary segmentation for the analysis of array-based DNA copy number data. Biostatistics 5: 557-572.

38. Charchar FJ, Kaiser M, Bingham AJ, Fotinatos N, Ahmady F, et al. (2010) Whole genome survey of copy number variation in the spontaneously hypertensive rat: relationship to quantitative trait loci, gene expression, and blood pressure. Hypertension 55: 1231-1238.

39. Ashburner M, Ball CA, Blake JA, Botstein D, Butler H, et al. (2000) Gene ontology: tool for the unification of biology. The Gene Ontology Consortium. Nat Genet 25: 25-29.

40. Kanehisa M, Goto S, Furumichi M, Tanabe M, Hirakawa M (2010) KEGG for representation and analysis of molecular networks involving diseases and drugs. Nucleic Acids Res 38: D355-D360.

41. Huang da W, Sherman BT, Lempicki RA (2009) Systematic and integrative analysis of large gene lists using DAVID bioinformatics resources. Nat Protoc 4: $44-57$.

42. Iafrate AJ, Feuk L, Rivera MN, Listewnik ML, Donahoe PK, et al. (2004) Detection of large-scale variation in the human genome. Nat Genet 36: 949951.

43. Graubert TA, Cahan P, Edwin D, Selzer RR, Richmond TA, et al. (2007) A high-resolution map of segmental DNA copy number variation in the mouse genome. PLoS Genet 3: e3.

44. Barrett T, Troup DB, Wilhite SE, Ledoux P, Rudnev D, et al. (2009) NCBI GEO: archive for high-throughput functional genomic data. Nucleic Acids Res 37: D885-D890. 